\title{
Editorial
}

\section{Pathogenesis of Bone Diseases: The Role of Immune System}

\author{
Giacomina Brunetti, ${ }^{1}$ Giorgio Mori, ${ }^{2}$ Patrizia D'Amelio, ${ }^{3}$ and Roberta Faccio ${ }^{4}$ \\ ${ }^{1}$ Section of Human Anatomy and Histology, Department of Basic Medical Sciences, Neuroscience and Sense Organs, \\ University of Bari, 70124 Bari, Italy \\ ${ }^{2}$ Department of Clinical and Experimental Medicine, University of Foggia, 71100 Foggia, Italy \\ ${ }^{3}$ Section of Gerontology and Bone Metabolism Diseases, Department of Medical Science, University of Torino, 10126 Torino, Italy \\ ${ }^{4}$ Department of Orthopedics, Washington University School of Medicine, St. Louis, MO 63110, USA
}

Correspondence should be addressed to Giacomina Brunetti; giacomina.brunetti@uniba.it

Received 15 February 2015; Accepted 15 February 2015

Copyright ( 2015 Giacomina Brunetti et al. This is an open access article distributed under the Creative Commons Attribution License, which permits unrestricted use, distribution, and reproduction in any medium, provided the original work is properly cited.

Bone is a metabolically active tissue that undergoes continuous remodeling by two sequential events, bone formation and resorption. These events are strongly linked and tightly regulated to maintain skeletal homeostasis. The bone cells responsible for the dual events include the bone-resorbing cells, the osteoclasts, arising from monocyte-macrophage precursors, and the bone forming cells, the osteoblasts, having a mesenchymal origin. Immune and bone cell activities are linked by several pathways [1] and the former can promote bone building or destruction. Further, immune cells can be involved in the mineralization process occurring in extra-skeletal sites. In this special issue different authors highlighted these items both through research articles and reviews.

In detail, interaction between osteoblast precursors, the mesenchymal stem cells (MSCs) [2], and immune cells during fracture repair acts as one of the key factors governing successful bone healing. Additionally, bone damage following immune deregulation may be local as in arthritis and periodontal disease (PD) or systemic as in osteoporosis [3] and osteotropic cancers $[4,5]$. It could be multifactorial and thus due to genetic modifications (i.e., Gaucher disease) as well as to lipopolysaccharide- (LPS-) mediated release of inflammatory cytokines (i.e., PD, osteomyelitis, and arthritis), and so forth. New insights suggest that, in immune-mediated bone diseases, bone resorption active phases are characterized by increased levels of immunoreceptor tyrosine-based activation motifs (ITAMs); these molecules together with OSCAR could be indicative of disease progression. Further, osteotropic cancer-related immune alterations showed distinct immune cell phenotype as observed in chronic myeloid leukemia, multiple myeloma, and bone metastatic solid tumors.

State-of-the-art and new mechanisms are clearly described in this special issue; they can be useful for the identification of new therapeutic targets and bone disease markers.

Giacomina Brunetti Giorgio Mori

Patrizia D'Amelio Roberta Faccio

\section{References}

[1] G. Mori, P. D’Amelio, R. Faccio, and G. Brunetti, "The interplay between the bone and the immune system," Clinical and Developmental Immunology, vol. 2013, Article ID 720504, 16 pages, 2013.

[2] G. Mori, M. Centonze, G. Brunetti et al., "Osteogenic properties of human dental pulp stem cells," Journal of Biological Regulators and Homeostatic Agents, vol. 24, no. 2, pp. 167-175, 2010.

[3] P. D’Amelio, A. Grimaldi, S. di Bella et al., "Estrogen deficiency increases osteoclastogenesis up-regulating T cells activity: a key mechanism in osteoporosis," Bone, vol. 43 , no. 1, pp. 92-100, 2008 . 
[4] G. Brunetti, G. Colaianni, M. F. Faienza, S. Colucci, and M. Grano, "Osteotropic cancers: from primary tumor to bone," Clinical Reviews in Bone and Mineral Metabolism, vol. 11, no. 3-4, pp. 94-102, 2013.

[5] I. Roato, "Interaction among cells of bone, immune system, and solid tumors leads to bone metastases," Clinical and Developmental Immunology, vol. 2013, Article ID 315024, 7 pages, 2013. 


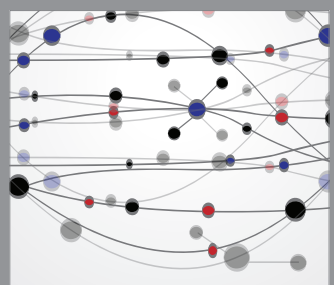

The Scientific World Journal
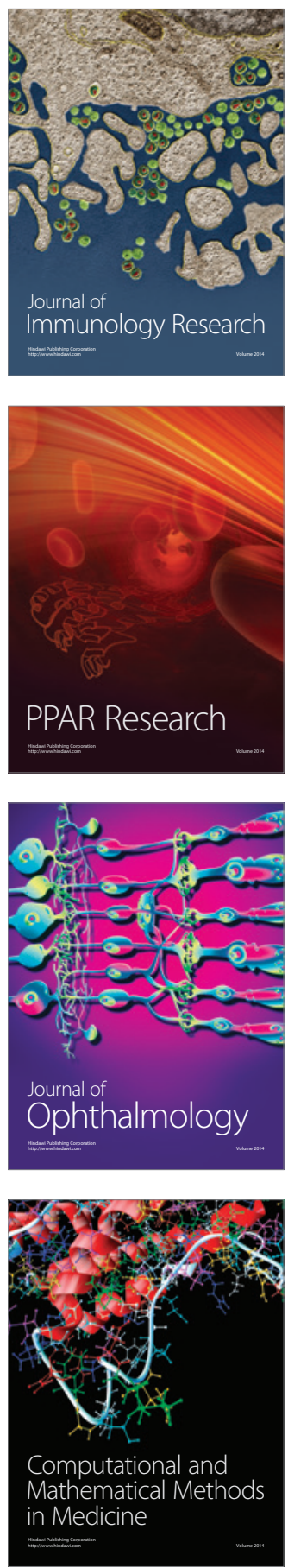

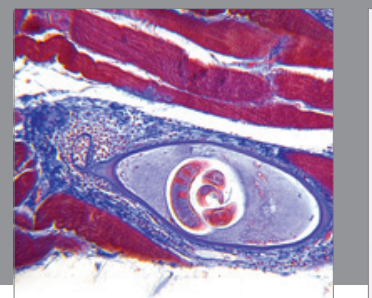

Gastroenterology

Research and Practice
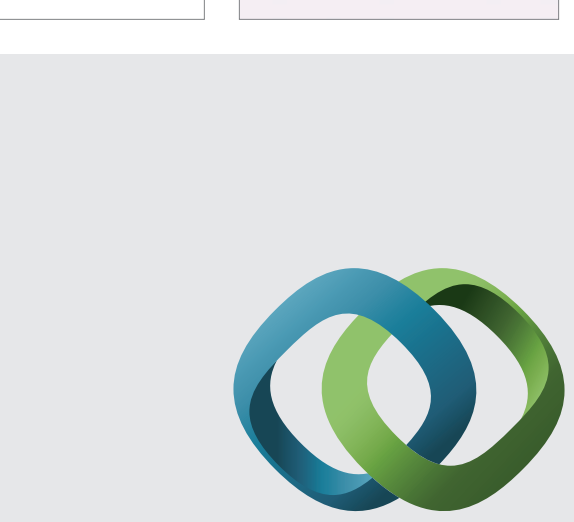

\section{Hindawi}

Submit your manuscripts at

http://www.hindawi.com
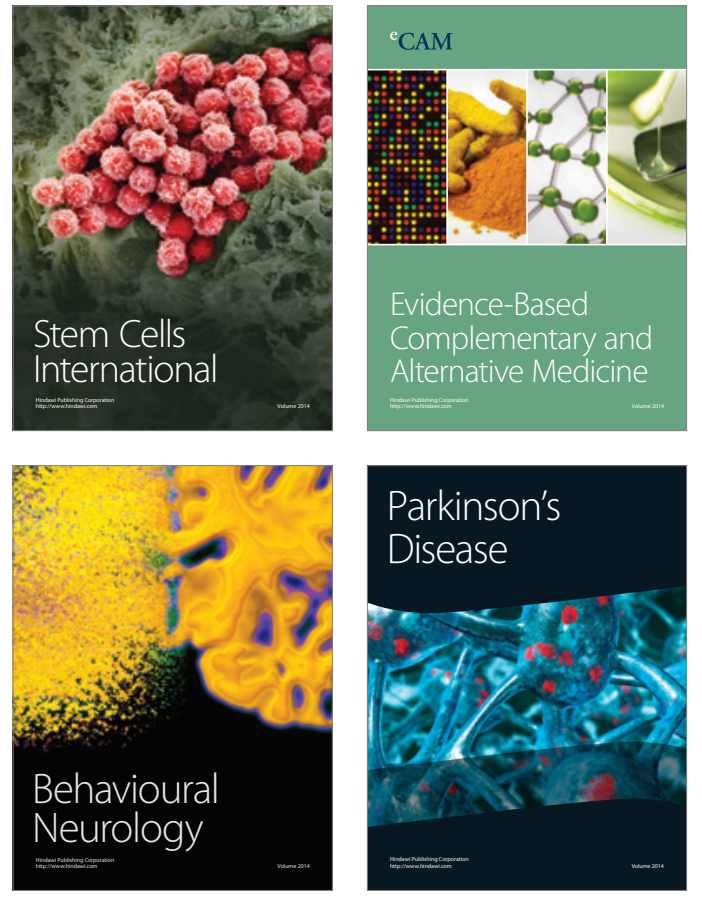
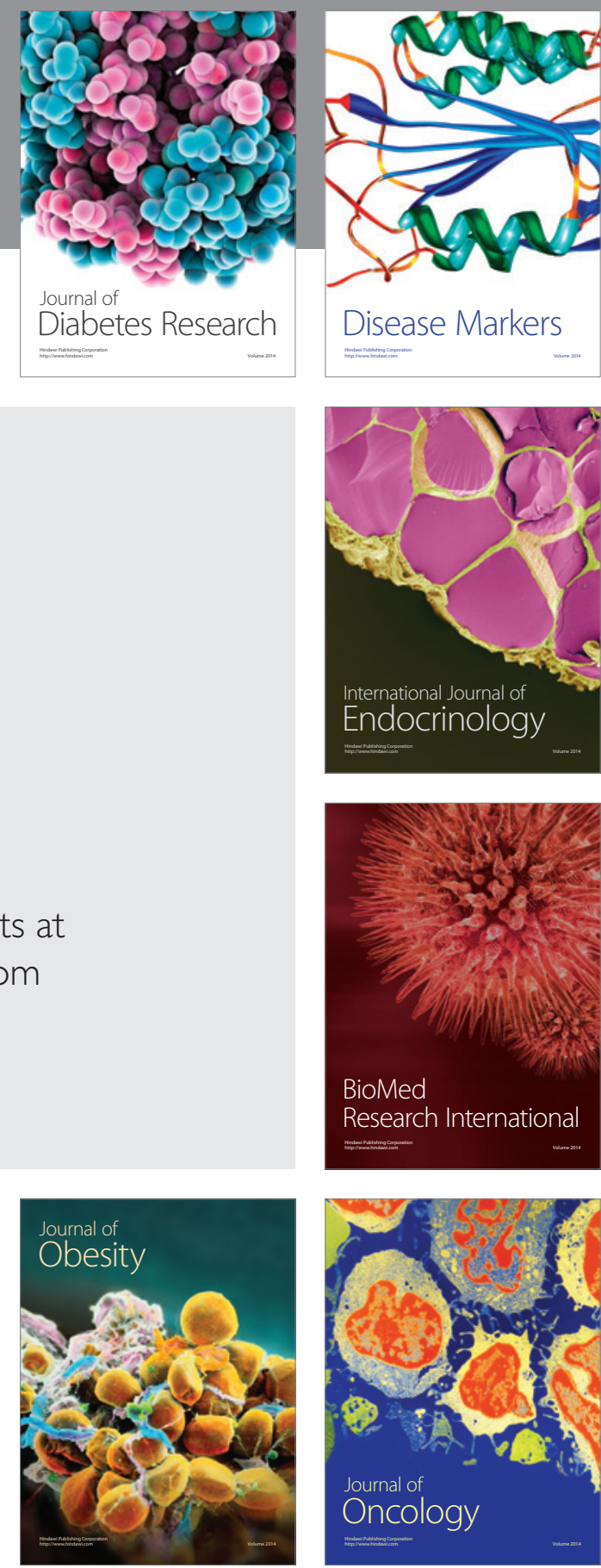

Disease Markers
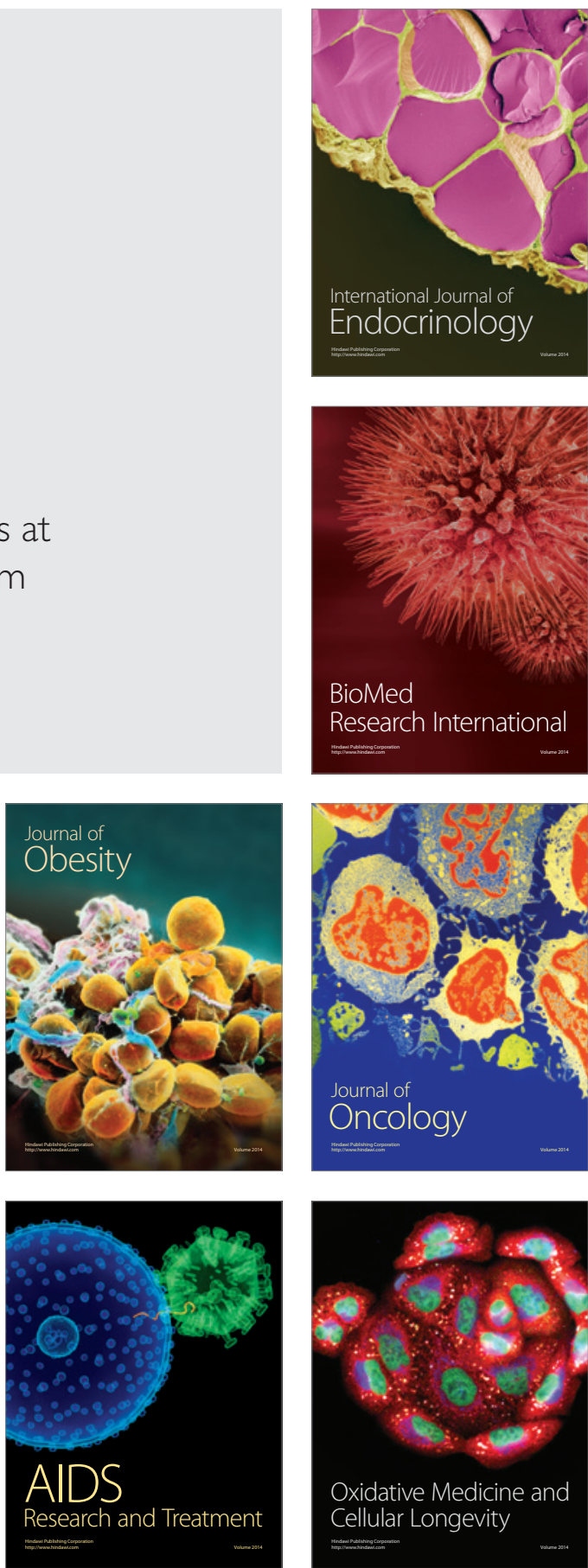